\title{
Sintomas depressivos e pré-frontais em idosos institucionalizados e não
}

\section{institucionalizados}

\author{
Depressive and prefrontal symptoms in institutionalized and non-institutionalized elderly \\ Síntomas depresivos y prefrontales en ancianos institucionalizados y no institucionalizados
}

Recebido: 25/07/2021 | Revisado: 30/07/2021 | Aceito: 05/08/2021 | Publicado: 10/08/2021

Olívia Dayse Leite Ferreira

ORCID: https://orcid.org/0000-0001-5298-8805 Centro Universitário Uniesp, Brasil

E-mail: oliviadayse@yahoo.com.br

Denise Pereira Reinaldo

ORCID: https://orcid.org/0000-0003-1022-4051 Centro Universitário Uniesp, Brasil

E-mail: denise_pereira01@ @otmail.com

Maria Denise Leite Ferreira

ORCID: https://orcid.org/0000-0001-8156-3443 Centro Universitário Uniesp, Brasil

E-mail: denisecaiana@yahoo.com.br

João Carlos Alchieri

ORCID: https://orcid.org/0000-0002-4150-8519 Universidade Federal do Rio Grande do Norte, Brasil E-mail: jcalchieri@gmail.com

\begin{abstract}
Resumo
O envelhecimento é um processo de desenvolvimento que provoca mudanças físicas, cognitivas, comportamentais e socioemocionais. O objetivo do presente trabalho consistiu em verificar as associações entre os sintomas pré-frontais e os sintomas depressivos em idosos institucionalizados e não institucionalizados. Participaram 206 pessoas, de ambos os sexos, com idade acima de 60 anos, sendo 109 indivíduos não institucionalizados e ativos da comunidade e 97 longevos residentes em instituições de longa permanência. Os participantes responderam ao Miniexame do Estado Mental (MEEM), a Escala de Depressão Geriatra (EDG-15), ao Inventário de Sintomas Pré-frontais (ISP-16) e um questionário sociodemográfico. Os resultados revelaram que os idosos institucionalizados exibiu maior média de idade (77 anos; $\mathrm{DP}=8,3$ ); a maioria possuía pouca escolaridade, eram, predominantemente, solteiros e sedentários. Apresentaram, também, mais déficits cognitivos (MEEM), sintomas subclínicos de depressão e pontuação maior no ISP-16, quando comparado aos não institucionalizados. Nos grupos, houve correlações positivas moderadas, estatisticamente significantes entre o ISP-16 e a EDG-15 ( $p<0,001)$ e negativa entre a escolaridade e o ISP-16 ( $\mathrm{p}<0,01)$. A análise de regressão múltipla corroborou o papel dos sintomas depressivos como um preditor significativo da variância dos sintomas pré-frontais e o papel da escolaridade como variável de confusão. Apesar das diferenças entre os contextos de moradia, aspectos sociodemográficos e estilo de vida, os sintomas depressivos estão as sociados aos pré-frontais, nos grupos, de forma semelhante, visto que regulações emocionais, comportamentais e cognitivas estão interligadas.
\end{abstract}

Palavras-chave: Depressão; Sintomas comportamentais; Instituição de longa permanência para idosos.

\begin{abstract}
Aging is a development process that leads to physical, cognitive, behavioral and socioemotional changes. This paper aims to verify association between prefrontal and depressive symptoms in older adults, institutionalized or not. 206 people, from both sexes and aged above 60 years, took part in this study, being 109 of them non-institutionalized and community active and 97 residents in long-term care institutions. Participants answered to the Mini-Mental State Examination (MMSE), the Geriatric Depression Scale (GDS-15), the Prefrontal Symptoms Inventory (PSI-16) and a sociodemographic questionnaire. Results revealed that residents in long-term care institutions group has exhibited higher age average (77 years; $\mathrm{SD}=8,3$ ); being the individuals mostly low schooled, single and sedentary. Also, those individuals presented more cognitive deficits (MMSE), subclinical symptoms of depression and higher score in ISP16 compared to non-institutionalized group. In both groups, there were moderate positive correlations, statistically significant between ISP-16 and GDS-15 ( $\mathrm{p}<0,001)$, and negative between schooling and ISP-16 ( $<<0.01)$. A multiple regression analysis confirmed the role of depression symptoms as a significative predictor of prefrontal symptoms variance and the role of schooling as a confusion variant. Despite the differences between habitation contexts, sociodemographic aspects and lifestyle, depressive symptoms are associated to prefrontal on a similar way in both groups, since emotional, behavioral and cognitive regulations are interconnected.
\end{abstract}

Keywords: Depression; Behavior symptoms; Homes for the aged. 


\section{Resumen}

El envejecimiento es un proceso de desarrollo que provoca cambios físicos, cognitivos, conductuales y socioemocionales. El objetivo de este estudio fue verificar las asociaciones entre síntomas prefrontales y síntomas depresivos en ancianos institucionalizados y no institucionalizados. Participaron un total de 206 personas, de ambos sexos, mayores de 60 años, 109 no institucionalizados y 97 longevos residentes en instituciones de larga estancia. Los participantes completaron el Mini Examen del Estado Mental (MMSE), la Escala de Depresión Geriátrica (EDG-15), el Inventario de Síntomas Prefrontales (ISP-16) y un cuestionario sociodemográfico. Los resultados revelaron que el GI tenía una edad media más alta $(77$ años; $\mathrm{DE}=8,3)$; la mayoría tenía poca educación, eran predominantemente solteros y sedentarios. También tenían más déficits cognitivos (MMSE), síntomas subclínicos de depresión y puntuaciones ISP-16 más altas en comparación con el INB. En los grupos, hubo correlaciones positivas moderadas, estadísticamente significativas entre ISP-16 y EDG-15 ( $p<0,001)$ y negativas entre educación e ISP-16 (p <0,01). El análisis de regresión múltiple apoyó el papel de los síntomas depresivos como un predictor significativo de la varianza de los síntomas prefrontales y el papel de la educación como variable de confusión. A pesar de las diferencias entre los contextos habitacionales, los aspectos sociodemográficos y el estilo de vida, los síntomas depresivos se asocian a los síntomas prefrontales, en grupos, de manera similar, ya que las regulaciones emocionales, conductuales y cognitivas están interconectadas.

Palabras clave: Síntomas depresivos; Síntomas de comportamiento; Hogares para ancianos.

\section{Introdução}

O envelhecimento pode ser entendido como um processo natural com diminuição progressiva da reserva funcional dos indivíduos e mudanças em indicadores de saúde, como funcionalidade, cognição e função psíquica. Trata-se de um fenômeno comum na maioria das sociedades e engloba os aspectos da senescência, as mudanças nos papéis sociais e a adaptação dos indivíduos às alterações do desenvolvimento (Santos et al, 2019).

Estima-se que dois bilhões de idosos viverão no mundo em 2050 e, até 2025, o Brasil será o sexto maior país no mundo em número desta população (Organização Mundial da Saúde, 2015). Aliado a esse crescimento do envelhecimento populacional e ao aumento da expectativa de vida, há uma elevação na taxa de doenças crônicas e doenças degenerativas que influenciam os membros da família a procurarem por Instituições de Longa Permanência para Idosos (ILPI).

As ILPI têm por objetivo que os idosos vivam de forma coletiva, usufruindo de hotelaria e cuidados hospitalares, além de atividades de lazer e ações interdisciplinares. A maioria das ILPI brasileiras é de pequeno porte (menos de 30 residentes por unidade), funcionando como prestador de serviços de saúde e como acomodação para aqueles que não têm renda nem domicílio. Essas instituições podem ser governamentais ou não e surgiram para prestar atendimento integral a idosos, cuja atenção familiar ou a permanência destes em seus lares não seja possível (Scherrer Júnior et al., 2019).

As ILPI têm por finalidade cuidar dos idosos, prestando assistência social e de saúde através do suporte às suas necessidades, que possibilita qualidade de vida e cuidados paliativos. Entretanto, essas instituições enfrentam problemas relacionados a recursos humanos, físicos e financeiros, a exemplo da falta de profissionais qualificados, atividades físicas, ocupacionais e funcionais. Além disso, tem dificuldades em cumprirem as diretrizes mundiais voltadas à atenção da saúde do idoso, bem como lidar com as limitações de cada idoso institucionalizado (Alves, Menezes, Felzemburg, Silva, \& Amaral 2017).

Idosos institucionalizados têm um perfil específico e particular, caracterizado por sedentarismo, perda de autonomia, ausência de visitas familiares e apoio financeiro insuficiente, entre outros fatores que afetam diretamente a independência. Perdas cognitivas também foram observadas em pesquisas recentes. Santos et al. (2019) detectaram, em uma amostra de 96 idosos institucionalizados, que 63 deles sofreram perdas cognitivas, sejam leves, moderadas ou graves.

Estudos apontam que a institucionalização tende a acelerar a dependência para a realização das atividades básicas da vida diária (ABVD), que são um indicador da capacidade funcional dos idosos no ambiente físico e social (Pagotto, Silva, Pereira, \& Santos, 2016). Na pesquisa de Imaginário, Machado, Rocha, Antunes e Martins (2017) sugeriu-se que as ABVD e as atividades instrumentais da vida diária (AIVD) explicam cerca de $49 \%$ da variância existente do estado mental dos idosos 
institucionalizados. Ou seja, diante do comprometimento funcional existe maior risco de déficits cognitivos. As ABVD são aquelas ligadas ao autocuidado, tais como banhar-se, vestir-se e alimentar-se. Já as AIVD estão relacionadas às ações mais complexas, como a participação social, que abrange o ato de fazer compras, usar o telefone, dirigir e usar meios de transporte coletivo.

Em um estudo longitudinal Jerez-Roig et al. (2016) observaram que, ao longo de dois anos, o declínio funcional nos idosos institucionalizados foi de 50\%, principalmente nas atividades que envolviam alimentar-se, vestir-se e andar. Além disso, a diminuição da capacidade funcional pode levar ao aparecimento da depressão, uma vez que a vida social e as atividades prazerosas se encontram limitadas. Scherrer Júnior et al. (2019) apontam que os sinais de depressão alteraram negativamente a qualidade de vida, principalmente nos domínios autonomia, atividades presentes, passadas e futuras e participação social. Os idosos dependentes para a realização das ABVD apresentaram alteração significante na qualidade de vida.

A depressão é comum em idosos, sendo considerado um grande problema de saúde pública, devido à sua morbilidade e excesso de mortalidade. Sintomas depressivos subclínicos, mesmo na ausência da patologia, estão associados a deficiências funcionais comparáveis ou piores do que aqueles com doenças cardíacas, artrite e diabetes. Os níveis subclínicos de sintomas depressivos afetam $10 \%$ a $15 \%$ dos idosos ativos da comunidade e elevam-se entre os residentes em ILPI (Mohebbi et al, 2019).

A depressão em idosos tem sido identificada como um possível fator de risco para a Doença de Alzheimer (DA) (Wang, Yip, Lu, \& Yeh, 2017). O processo pelo qual a depressão pode afetar esse risco não foi bem estabelecido na literatura. Porém, a depressão está associada a alterações cerebrais, incluindo atrofia do hipocampo e disfunções metabólicas no córtex orbitofrontal, o qual está relacionado ao córtex pré-frontal (CPF).

O CPF é compreendido como uma região de associação interligada com uma multiplicidade de regiões corticais e subcorticais, permitindo que o sistema seja orquestrado através de mecanismos de controle, organização e coordenação. Postula-se que a cognição, a emoção e o comportamento social estejam relacionados a essa estrutura, sendo comandadas por áreas independentes e interligadas entre si (Fonseca, Yokomizo, Bottino, \& Fuentes 2016).

De acordo com o estudo desenvolvido por Klojčnik, Kavcic e Vukman (2017) as dificuldades no funcionamento executivo e na memória visuoespacial são os melhores preditores de depressão em idosos. Esta cognição prejudicada poderia estar relacionada à alteração na secreção de cortisol, o qual exerce seus efeitos no cérebro, por meio de dois receptores nucleares diferentes, ambos abundantemente expressos no hipocampo e no CPF, áreas cerebrais críticas para a memória e as Funções Executivas (FE).

Estudos foram desenvolvidos com o objetivo de compreender a associação entre sintomas depressivos e alterações nas funções do CPF em idosos, principalmente no que se referem às FEs. No estudo desenvolvido por Santorelli e Ready (2015), procurou-se investigar a associação entre a alextimia (dificuldades em descrever emoções e sentimentos) com as FE em adultos mais velhos com sintomas depressivos. Os resultados revelaram que uma maior alextimia foram associados com um desempenho fraco nas FEs. Philippot e Agrigoroaei (2017) realizaram testes de funcionamento executivo (teste de Stroop, teste de trilha e teste de fluência), além de questionários para pensamento repetitivo e depressão. Observaram-se correlações positivas entre pensamento repetitivo abstrato e humor depressivo, e entre pensamento repetitivo concreto e funcionamento executivo. Além disso, uma correlação negativa foi observada entre humor depressivo e funcionamento executivo.

Embora o conceito de FEs seja bastante utilizado, existe uma grande variação na forma como este constructo é definido. Não há uma definição genérica sobre as FEs, bem como não há consenso sobre as habilidades que esse constructo abrange. Um dos modelos defendido por Zelazo, Qu e Muller em 2005 enfatiza aspectos tantos cognitivos como socioafetivos, classificando-as em processos executivos "frios" (cold) e "quentes" (hot). Os componentes "frios" envolve os aspectos mais lógicos e cognitivos, como o raciocínio lógico e abstrato, planejamento, resolução de problemas e memória de trabalho. Por 
outro lado, os processos "quentes" estão mais relacionados com os aspectos emocionais, como a regulação do afeto, da motivação e do próprio comportamento social, tomada de decisão, experiência de recompensa, interpretações pessoais e julgamento moral (Uehara, Charchat-Fichman, \& Landeira-Fernandez, 2013).

Percebe-se que na literatura há um consenso ao afirmar que a depressão interfere no funcionamento das FEs dos idosos. Entretanto, são escassas as pesquisas que investigam as associações entre os sintomas subclínicos de depressão e os problemas comportamentais (de ordem executiva, atencional, motivacional e emocional) ocasionados pela vulnerabilidade do $\mathrm{CPF}$ ao envelhecimento.

Portanto, faz-se necessário pesquisar como os sintomas depressivos estão associados aos sintomas pré-frontais autorelatados subjetivamente, no processo de envelhecimento, considerando dois contextos de moradia distintos (comunidade e ILPI). Uma melhor apreensão das dificuldades funcionais e incapacitantes apresentadas por essa população se faz importante para o planejamento de ações que melhorem a qualidade de vida, que visem minimizar o impacto causado pela articulação desses sintomas.

\section{Método}

\section{Participantes}

Constituiu-se como um estudo transversal e quantitativo, realizadas com idosos residentes na região da grande João Pessoa, situada no estado da Paraíba. Foi composto por voluntários, de ambos os sexos, com idade acima de 60 anos, subdivididos em dois grupos, um não institucionalizados e ativos da comunidade, chamado de GNI, recrutados em centros de convivências e espaços recreativos e o segundo grupo composto por idosos residentes em cinco ILPI governamentais e não governamentais, denominado de GI. Foram inclusos na amostra os idosos, idade $\geq 60$ anos, que apresentavam condições favoráveis para a compreensão dos questionários, segundo MEEM de 19 pontos ou mais para alfabetizados e dados do prontuário. Foram excluídos os idosos que não aceitaram participar do estudo, que apresentavam rebaixamento do nível de consciência, cognição prejudicada, abuso de substâncias psicoativas autorelatada, alterações visuais ou auditivas identificáveis que pudessem intervir na pesquisa e que não falavam ou entendiam a língua portuguesa.

\section{Instrumentos}

O MEEM foi desenvolvido nos Estados Unidos composto por cinco dimensões (orientação, atenção, concentração, memória, cálculo, linguagem e práxis) baseadas em análise teórica e prática clínica. É o teste mais utilizado para avaliar a função cognitiva por ser rápido (em torno de 10 minutos), de fácil aplicação, não requerendo material específico. Deve ser utilizado como instrumento de rastreamento não substituindo uma avaliação mais detalhada, pois, apesar de avaliar vários domínios, não serve como teste diagnóstico, mas sim pra indicar funções que precisam ser investigadas. Foi utilizada a adaptação elaborada por Brucki, Nitrini, Caramelli, Bertolucci, e Okamoto (2003), com escore máximo de 30 pontos.

A Escala de Depressão Geriátrica (EDG) é um instrumento de auto relato em formato dicotômico (sim ou não), comumente usado para avaliação clínica de depressão em adultos mais velhos. Neste estudo utilizou-se a versão 15 (Alvarenga, Oliveira, \& Faccenda, 2012). O ponto de corte adotado, no valor maior ou igual a seis, corresponde a índices adequados de sensibilidade $(85,4 \%)$ e especificidade $(73,9 \%)$ para rastreio de sintomas depressivos. A consistência interna da escala apresenta alfa de Cronbach de 0,81 .

Já o Inventário de Sintomas Pré-Frontais (ISP) trata-se de um instrumento de origem espanhola (Ruiz-Sánchez de León, Pedrero-Pérez, Lozoya-Delgado, Llanero-Luque, Rojo-Mota, \& Puerta-García, 2012) composto por 46 itens $(\alpha>0,94)$, com propriedades psicométricas também adequadas para a versão abreviada, contendo 20 itens $(\alpha>0,89)$, respondidas em uma escala do tipo Likert ( 0 a 4 pontos). Composto na sua versão original por três subescalas, teoricamente independentes, a 
primeira delas avalia os problemas comportamentais, incluindo problemas motivacionais, problemas de controle executivo e problemas de atenção (composta por 12 itens); a segunda envolve os problemas nas condutas sociais (composta por quatro itens) e a terceira os problemas no controle emocional (composta por quatro itens).

Na versão para o português brasileiro (Ferreira, Barbosa, \& Alchieri, 2020), entretanto, as evidências de validade demonstraram índices satisfatórios para um modelo unifatorial ( $\Omega$ de 0,83 e $\alpha=0,80$ ), contendo 16 itens (versão utilizada na presente pesquisa), ou seja, não apresentou divisão entre as subescalas. O objetivo do instrumento é explorar comportamentos da vida cotidiana que se relacionam com alterações no córtex pré-frontal, na esfera comportamental, cognitiva e emocional de forma qualitativa.

\section{Procedimentos}

O estudo foi aprovado pelo comitê de ética em pesquisa do Hospital Universitário Onofre Lopes da Universidade Federal do Rio Grande do Norte (CAEE 50929115.7.0000.5292), e a coleta de dados foi realizada no período de fevereiro a dezembro de 2019, por psicólogos e estudantes de psicologia em cinco ILPI, centros de convivência e espaços recreativos para idosos na grande João Pessoa-PB. Realizou-se uma investigação por meio dos prontuários dos usuários nos ambientes, supracitados, e todos que a priori se enquadravam nos critérios de inclusão e exclusão da pesquisa foram convidados a participarem. Em entrevista individual, em ambiente privado, na própria ILPI (exemplo, quarto, sala de atendimento, salão de recreação) e nas salas fornecidas pela equipe de psicologia e assistência social nos centros de convivência e espaços recreativos, os participantes foram informados sobre o estudo e solicitado o consentimento por escrito ou por meio da impressão digital antes de qualquer procedimento, em consonância com o que preconiza a Resolução n 466/2012. Nas etapas iniciais (entrevista e MEEM) cerca de 10 participantes não se mostraram aptos a participarem da pesquisa e não seguiram para as próximas etapas. Outros seis idosos desistiram em algumas das etapas e, portanto, não foram incluídos na amostra total.

A amostra foi composta por 206 idosos, alocados no grupo definido pela sua condição, sendo que cada atendimento durou 25 minutos, quando se realizou uma entrevista estruturada elaborada coletar dados, demográficos, físicos e mentais, bem como uma avaliação breve do estado psíquico por meio do MEEM. Para avaliação dos sinais e sintomas de depressão utilizouse a EDG-15 e com o ISP-16 mensurou-se os sintomas pré-frontais dos participantes.

\section{Análise estatística}

Os dados individuais foram agrupados em planilha específica do software IBM SPSS Statistics (versão 22.0), analisados de formas descritiva, comparativa e associativa, conforme os objetivos da pesquisa. Foram realizados testes de normalidade da distribuição dos resultados (teste de Kolmogorov-Smirnov), identificando-se que as variáveis atendiam aos critérios pré-estabelecidos da pesquisa.

Na comparação dos grupos nas variáveis sociodemográficas e clínicas (MEEM, EDG-15 e ISP-16), recorreu-se ao teste $\mathrm{t}$ Student para os valores médios e o teste do qui-quadrado para as variáveis categóricas, sempre ao nível de significância de 5\%. Foi calculado o d de Cohen para se obter o tamanho do efeito das diferenças identificadas nas análises comparativas, considerando-se: d próximo a $0,20=$ efeito pequeno ou fraco, d próximo a $0,50=$ efeito médio ou moderado, d maior que $0,80=$ efeito grande.

Em ambos os grupos, para compreender a associação entre os sintomas pré-frontais e depressivos recorreu-se ao teste de correlação r Pearson e posteriormente, produziu-se a análise de regressão múltipla (método hierárquico) para examinar se as variáveis sociodemográficas e a sintomatologia depressiva, em conjunto, predizem significativamente os sintomas pré-frontais. Considerou-se a idade, nível de escolaridade, sexo, tempo de institucionalização e sintomatologia depressiva (EDG), como variáveis independentes; e a sintomatologia pré-frontal (ISP), como variável dependente. 


\section{Resultados}

Nos dados sociodemográficos (Tabela 1), pode-se observar que o GNI possuía idade média de 71,3 anos (DP = 7,8), sendo a maioria do sexo feminino $(78 \%)$, casada $(42,1 \%)$ e com escolaridade acima de oito anos de estudo $(65,1 \%)$. Percebeuse também que $56,9 \%$ dos participantes relataram dificuldades na cognição, a maior parte praticava atividade física com frequência $(86,2 \%)$ e fazia uso de algum tipo de medicamento de forma contínua $(92,7 \%)$.

Já no GI, o tempo médio de institucionalização foi de 43 meses (DP = 55,3), com idade média dos participantes de 77 anos (DP $=8,3)$, a maioria do sexo feminino $(67 \%)$, solteira $(36,1 \%)$ e escolaridade de até quatro anos $(59,8 \%)$. Os participantes deste grupo também relataram poucas queixas subjetivas de déficits cognitivos (62,9\%) e fazer uso de medicamentos de forma contínua (90,7\%). Por outro lado, a maioria não praticava atividade física com frequência (77,3\%).

$\mathrm{Na}$ comparação entre os grupos, por meio do teste t de Student e do Qui quadrado, pode-se observar que houve diferenças estatisticamente significantes nas variáveis: idade $(\mathrm{t}(204)=-5,60 ; \mathrm{p}<0,001 ; \mathrm{d}=0,69 ; 95 \%$ IC [-7,91; -348]), escolaridade $(\chi 2=29,1, \mathrm{p}<0,001)$, estado civil $(\chi 2=51,3 ; \mathrm{p}<0,001)$ e na prática de atividade física $(\chi 2=84,2 ; \mathrm{p}<0,001)$.

Tabela 1: Características sociodemográficos dos grupos de idosos institucionalizados e ativos da comunidade.

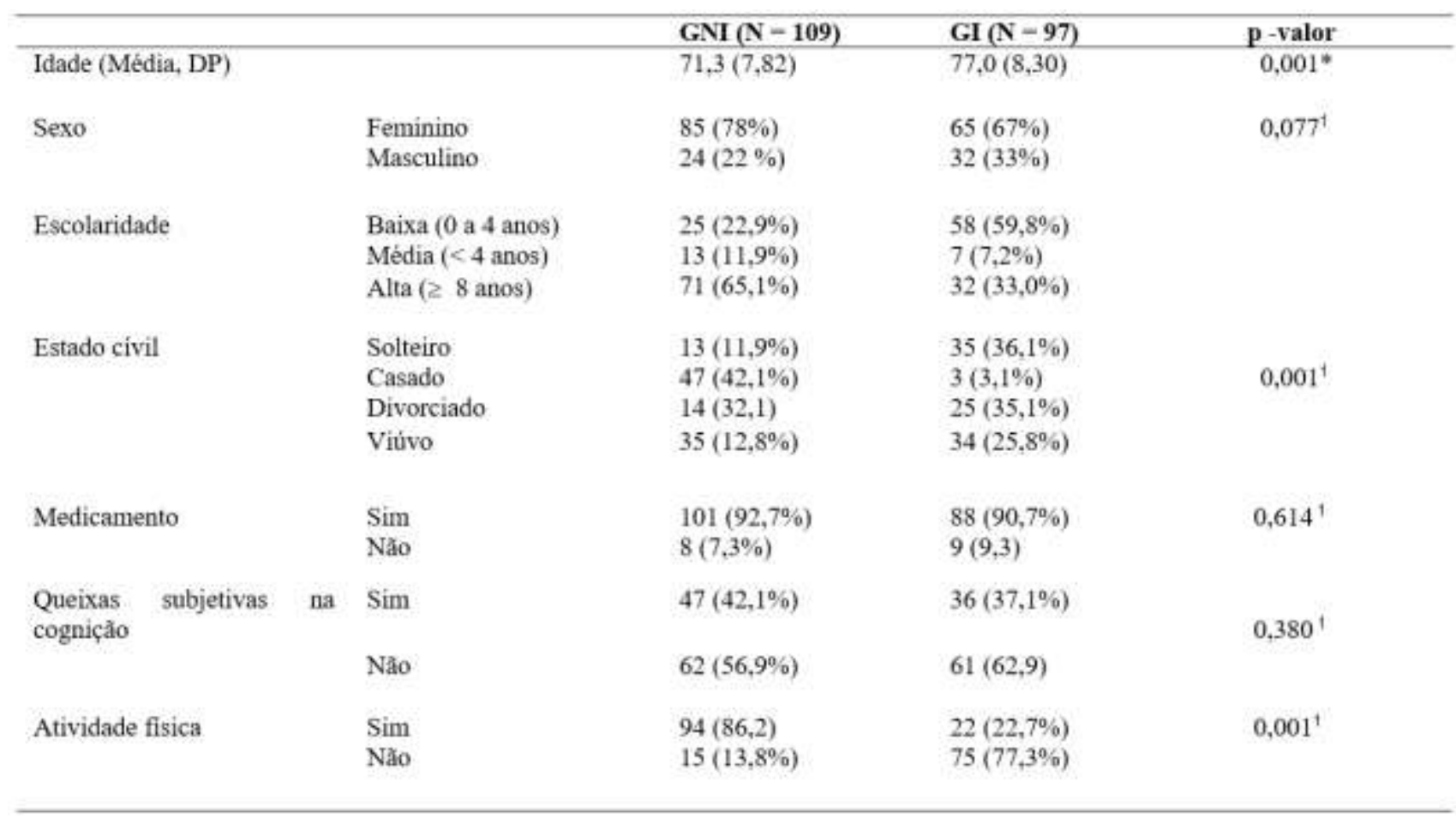

Nota: GNI = grupo não institucionalizado; GI=grupo institucionalizado; *Teste t de Student para amostras independentes (comparação de médias entre os dois grupos); 1 Qui-quadrado (comparação entre os dois grupos). Fonte: Autores.

Na comparação entre os grupos com relação às variáveis clínicas (Tabela 2), pode-se perceber que houve diferenças estatisticamente significantes entre o GNI e o GI no MEEM, na EDG-15 e na ISP-16, com tamanho de efeito (d de Cohen) variando de moderado a alto. O GI apresentou o pior desempenho no MEEM e os maiores sintomas de depressão e de dificuldades pré-frontais. 
Tabela 2: Características clínicas e comparação entre os grupos de idosos institucionalizados e ativos da comunidade.

\begin{tabular}{|c|c|c|c|c|}
\hline \multirow{3}{*}{ Variáveis } & \multicolumn{2}{|c|}{ Grupos } & & \\
\hline & \multirow{2}{*}{$\frac{\text { GNI (109) }}{M(D P)}$} & \multirow{2}{*}{$\frac{\text { GI (97) }}{M(D P)}$} & \multicolumn{2}{|c|}{ Comparação estatística } \\
\hline & & & Teste $t$ & d de Cohen \\
\hline MEEM & $25,3(4,3)$ & $21,2(8,5)$ & $4,39 * * *$ & 0,60 \\
\hline GDS-15 & $2,9(2,3)$ & $4,3(3,1)$ & $-3,60 * * *$ & 0,51 \\
\hline ISP-16 & $16,21(8,98)$ & $22,06(8,43)$ & $-4,80 * * *$ & 0,67 \\
\hline
\end{tabular}

Nota: MEEM: Mini Exame do Estado Mental; GDS: Inventário de Depressão Geriatrica; ISP: Inventário de Sintomas Préfrontais; Teste $\mathrm{t}$ de Student para amostras independentes (comparação de médias entre os dois grupos); *** $\mathrm{p}<0,001$. Fonte: Autores.

Em ambos os grupos, houve correlações positivas (moderada/forte) estatisticamente significantes entre a GDS-15 e o ISP-16, ou seja, quanto maior os sintomas depressivos, maior seriam os sintomas pré-frontais. Encontraram-se também correlações moderadas positivas, com significância estatística, entre o ISP-16 e a escolaridade nos dois grupos (GIN e GI), sinalizando que o desempenho no ISP-16 tem relação com a escolaridade (Tabela 3).

Não foram encontradas correlações estatisticamente significantes entre a variável de tempo de institucionalização, sintomas depressivos $(r=0,18, \mathrm{p}>0,05)$ e pré-frontais $(\mathrm{r}=0,14, \mathrm{p}>0,05)$.

Tabela 3: Correlações entre os sintomas pré-frontais, os sintomas depressivos e as variáveis sociodemográficas nos grupos de idosos institucionalizados e ativos da comunidade.

\begin{tabular}{|c|c|c|c|c|c|c|c|c|}
\hline \multirow{3}{*}{ Variáveis } & \multicolumn{7}{|c|}{ Grupos } & \\
\hline & \multicolumn{3}{|c|}{ GNI } & \multicolumn{5}{|c|}{ GI } \\
\hline & 1 & 2 & 3 & 4 & 1 & 2 & 3 & 4 \\
\hline \multicolumn{9}{|l|}{ 1. ISP } \\
\hline 2. GDS-15 & $0,55^{\text {** }}$ & & & & $0,45^{* *}$ & & & \\
\hline 3. Idade & 0,10 & 0,06 & & & 0,06 & $-0,02$ & & \\
\hline 4. Escolaridade & $-0,23^{*}$ & $-0,17$ & $-0,13$ & 1 & $-0,32^{* *}$ & $-0,05$ & $-0,15$ & 1 \\
\hline
\end{tabular}

Nota: GDS-15: Inventário de Depressão Geriátrica; ISP: Inventário de Sintomas Pré-frontais; Correlação r de Pearson; * $\mathrm{p}<0,05, * * \mathrm{p}<0,01, * * * \mathrm{p}<0,001$. Fonte: Os autores

Para compreender o poder preditivo dos sintomas depressivos nos sintomas pré-frontais, realizou-se uma regressão múltipla com o método hierárquico, controlando também certas variáveis sociodemográficas para ambos os grupos (GNI e GI) (Tabela 4).

Na primeira etapa, inseriram-se as variáveis: escolaridade, sexo, idade e tempo de institucionalização (para o GI). Já a pontuação total do GDS-15 entrou na segunda etapa da análise. As variáveis demográficas explicaram $8 \%$ da variância do ISP$16(\mathrm{~F}(3,93)=3,80 ; \mathrm{p}=0,01)$ para o GNI e cerca de $11 \%$ para o GI $(\mathrm{F}(4,92)=3,94 ; \mathrm{p}=0,01)$. Os sintomas depressivos explicaram $37 \%$ da variância adicional dos sintomas pré-frontais $(\mathrm{F}(1,92)=24,1 ; \mathrm{p}<0,001)$ no GNI e $27 \%$ no GI $(\mathrm{F}(1,91)=$ $21,1 \mathrm{p}<0,001)$. 
Tabela 4: Regressão múltipla hierárquica para testar o valor de predição dos sintomas depressivos em relação aos sintomas pré-frontais

\begin{tabular}{|c|c|c|c|c|c|c|c|c|}
\hline \multicolumn{5}{|c|}{ GNI } & \multicolumn{4}{|c|}{ GI } \\
\hline Variáveis & B & DP & $\boldsymbol{\beta}$ & $\Delta \mathbf{R}^{2}$ & B & DP & $\beta$ & $\Delta \mathbf{R}^{2}$ \\
\hline ISP-16 & & & & & & & & \\
\hline Passo 1 & & & & $0,08 *$ & & & & $0,11^{*}$ \\
\hline Constante & 15,85 & 8,43 & & & 25,9 & 8,64 & & \\
\hline NI & $-2,61$ & 1,01 & $-0,24^{*}$ & & $-2,79$ & 0,90 & $-0,30^{*}$ & \\
\hline Sexo & 3,62 & 2,07 & 0,16 & & $-0,77$ & 1,75 & $-0,04$ & \\
\hline Idade & 0,03 & 0,11 & 0,02 & & 0,03 & 0,09 & 0,03 & \\
\hline $\mathrm{TI}^{*}$ & 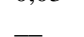 & . & 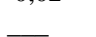 & & 0,03 & 0,01 & 0,20 & \\
\hline Passo 2 & & & & $0,37^{* * *}$ & & & & $0,27^{* * *}$ \\
\hline Constante & 23,6 & 6,65 & & & 19,5 & 7,87 & & \\
\hline GDS-15 & 2,08 & 0,30 & $0,54^{* * *}$ & & 1.09 & 0,23 & $0,41^{* * *}$ & \\
\hline
\end{tabular}

Nota: NI: nível de escolaridade; TI: Tempo de institucionalização; Modelo de regressão múltipla $(* \mathrm{p}<0,05$, **p<0,01, ***p<0,001); NI tratada como uma variável intervalar e sexo como binária. Fonte: Autores.

\section{Discussão}

O objetivo da presente pesquisa consistiu em verificar as associações entre os sintomas depressivos e os sintomas préfrontais em idosos institucionalizados e ativos da comunidade. Os resultados revelaram que, apesar das diferenças entre o GNI e GI, os sintomas depressivos estão associados aos pré-frontais, nos grupos, de forma semelhante, sendo o primeiro um preditor significativo da variância do segundo.

Os dois grupos estudados apresentaram diferenças importantes, com os participantes do GI exibindo idade superior aos dos indivíduos que residiam na comunidade (aproximadamente seis anos). A maioria possuía pouca escolaridade, eram, predominantemente, solteiros e sedentários. Exibiram, também, mais déficits cognitivos identificados pelo MEEM, sintomas subclínicos de depressão e pontuação alta no ISP-16, quando comparado ao GNI.

O perfil do GI vai ao encontro dos outros estudos brasileiros, em que se identificou maior frequência de idosos do sexo feminino, analfabetos, solteiros e sem filhos. Com relação à escolaridade, Azevedo et al. (2017) ressalta que quanto maior a idade, menores a oportunidade de estudo, visto que em décadas passadas como 1940 não precisava de muita instrução para conseguir emprego. Além disso, o contexto social do século passado não conduzia à escolarização do sexo feminino.

$\mathrm{Na}$ avaliação do estado mental, pode-se perceber que os idosos institucionalizados apresentaram desempenho inferior aos dos longevos ativos da comunidade corroborando os achados de Costa et al. (2021), onde o grupo de indivíduos não institucionalizados obteve média no MEEM de 22,11 contrapondo 17,03 no grupo dos institucionalizados. Concluiu-se que a baixa escolaridade e a institucionalização contribuem de forma crucial para a degradação cognitiva. Os mesmos apresentam maiores chances de desenvolver patologias que causam decadências físicas e psicológicas mais expressivas, que os não institucionalizados. Vale salientar, contudo, que a falta de adaptação transcultural, validação e normatização do MEEM no Brasil podem reproduzir falsos positivos e negativos.

Quanto aos sintomas de depressão, pode-se verificar que os grupos não atingiram o ponto de corte mínimo da escala para a identificação do transtorno (6 pontos), entretanto, como hipotetizado, o GI exibiu a maior média dos escores. No grupo dos idosos, um índice elevado de vulnerabilidade à depressão tem sido observado naqueles institucionalizados, talvez pelo isolamento social, a ausência dos familiares, as doenças físicas e a perda de autonomia.

No ISP-16, também se identificou diferenças estatisticamente significantes entre o GI e GNI. Os sintomas pré-frontais envolvem componentes que estão intrinsecamente relacionados: Motivação (impulso e interesse em iniciar um comportamento); Controle de execução (capacidade de desenvolver um plano, ter flexibilidade cognitiva e resolver problemas), Atenção (gerenciamento da atenção) e Controle emocional (labilidade emocional) (Kaster et al, 2018). 
Os idosos institucionalizados, portanto, apresentaram mais perdas cognitivas, comportamentais e labilidade emocional, as quais podem estar associadas à dependência e às dificuldades em realizar as ABVD e as AIVD. Segundo o que dizem Zimmermann et al. (2015) a restrição na execução de AIVD ou de lazer contribui para a diminuição das capacidades cognitivas. Deste modo, os estímulos intelectuais, recreativos e sociais, convergem para a manutenção mais saudável das funções cognitivas, a modificação da rotina diária no contexto institucional e a realização das atividades por outras pessoas levam à perda de habilidades e à falta de motivação para executar determinados comportamentos. Dentre as várias alterações fisiológicas decorrentes do processo de envelhecimento, as funções do sistema nervoso central, principalmente as de origem neuropsicológica, podem comprometer o bem-estar biopsicossocial.

Nos grupos, houve correlações positivas moderadas estatisticamente significantes, entre os sintomas depressivos e os sintomas pré-frontais. Independentemente do contexto de moradia, quanto maior forem os sintomas depressivos, maiores serão os problemas pré-frontais. O ISP-16 foi construído no intuito de mensurar os sintomas pré-frontais, considerando-se a integração dos processos cognitivos, emocionais e motivacionais, intrínsecos nas atividades da vida diária (Ruiz-Sánchez et al, 2012). As evidências científicas têm mostrado que os indivíduos não utilizam uma função independentemente das outras, assim sendo, não é de surpreender o fato dos aspectos afetivos interagirem com os cognitivos e comportamentais de forma positiva.

Do ponto de vista neuroanatômico, pode-se ressaltar que o CPF ventromedial e o CPF dorsolateral (CPF medial) estão implicados no processo de regulação emocional e fazem conexões estruturais com várias áreas-chave, como o córtex cingulado posterior, o hipocampo, bem como a amígdala, insula (rede de saliência), caudado e putamen (sistema de recompensa), córtex temporal médio (chave da teoria da mente), córtex cingulado anterior dorsal e CPF ventrolateral, envolvidos no controle inibitório e na atenção (Kaster et al, 2018; Steffens, Wang, Manning, e Pearlson, 2017). Portanto, as alterações de humor provocadas pelos sintomas depressivos podem interagir com as regulações emocional, cognitiva e comportamental.

Os resultados reforçam achados anteriores, como o desenvolvido por Murayama et al. (2019), no qual o baixo escore na categoria cálculos (memória de trabalho) do MEEM foi associado com altos escores no EDG-15 em idosos. No estudo de Otte, Wingenfeld, Kuehl, Richter, Regen, Piber, e Hinkelmann (2015), idosos com depressão maior exibiram pior desempenho na velocidade psicomotora, no funcionamento executivo, na aprendizagem verbal e na memória verbal.

Dentre as variáveis sociodemográficas, a escolaridade correlacionou-se negativamente de forma moderada com o ISP$16 \mathrm{em}$ ambos os grupos estudados. O ISP-16 consiste em um instrumento de auto-relato, que exige capacidade de julgamento e consciência dos comportamentos, portanto, quanto maior a escolaridade, melhor a percepção dos participantes. Estudos vêm evidenciando que, quanto mais elevada à escolaridade, maior a estimulação intelectual e a promoção de crescimentos axonal e sináptico, permitindo a formação de uma reserva cognitiva, que protege o cérebro de processos patológicos (Steffens et al, 2017).

A análise de regressão múltipla corroborou o papel dos sintomas depressivos como um preditor significativo da variância dos sintomas pré-frontais, tanto no GNI como no GI. Ou seja, independentemente da condição de moradia e variáveis sociodemográficas, os sintomas de depressão nos idosos podem ter impacto nos problemas cognitivos, comportamentais e emocionais que estão relacionados ao córtex pré-frontal. Além disso, ressaltou que a escolaridade exerceu influência nas respostas do ISP-16.

As pesquisas vêm enfatizando que os sintomas depressivos podem ser preditores de mudanças longitudinais na cognição. Nesta perspectiva, pode-se citar o estudo de Formánek et al. (2020) que realizaram uma pesquisa de coorte multinacional em 69.066 participantes avaliando funções cognitivas e sintomas depressivos por dois anos. Os participantes com sintomas depressivos crescentes tiveram o declínio cognitivo mais rápido, enquanto naqueles com sintomas depressivos 
decrescentes foram resguardados do declínio na cognição. Kiyoshige et al. (2019) concluíram em estudo longitudinal que detectar sintomas depressivos pode ser uma chave para prevenir o declínio das AIVD em pessoas na faixa dos 70 anos.

No presente estudo, pode-se sugerir que os sintomas depressivos nos idosos se configuraram como um importante preditor dos sintomas comportamentais, provocados por alterações do CPF. O ISP-16 interagiu com o EDG-15 de forma harmônica, demonstrando que o controle emocional é um importante componente do constructo geral de sintomas pré-frontais. Reforçam-se, assim, os achados da literatura que ressaltam as interações entre a regulação emocional, os aspectos cognitivos, motivacionais e atencionais (Ruiz-Sánchez et al, 2012).

Por fim, muito embora este estudo tenha trazido resultados interessantes sobre a articulação entre os sintomas préfrontais e depressivos em idosos, os mesmos devem ser interpretados com cautela, levando-se em consideração suas limitações. Eles sobrevêm de uma amostra não probabilística de um grupo de idosos residentes em ILPI de característica governamental e filantrópica, e de grupos da comunidade que visam o envelhecimento ativo. Além disso, os inventários utilizados (EDG-15 e ISP-16) necessitavam de autoconsciência e consciência dos outros. Estudos têm relatado que a anosognosia (falta de conscientização sobre os déficits) pode ser algo presente no processo de envelhecimento (Gerstenecker, Triebel, Eakin, Martin, \& Marson 2017). A presente pesquisa também não avaliou os sintomas depressivos dos idosos longitudinalmente. Alguns estudos tem revelado que à medida que as pessoas com depressão envelhecem, sua disfunção executiva se torna mais acentuada.

No que diz respeito à sua aplicabilidade, os achados permitem fornecer indícios sobre a saúde mental dos idosos e possibilitam pensar sobre ações promotoras de qualidade de vida pautadas nos aspectos psíquicos, cognitivos e sociais. Tanto as ILPI como os grupos de convivência para adultos mais velhos necessitam promover programas que estimulem a regulação emocional, a prevenção de declínio cognitivo e a melhoria da funcionalidade e da independência. Sugere-se, ainda, o seguimento de estudos com uma amostra mais representativa de adultos mais velhos, envolvendo principalmente participantes do sexo masculino e um maior nivelamento da escolaridade, visto que a mesma foi uma variável relevante para as respostas ao ISP-16.

\section{Conclusões}

A presente pesquisa destacou a importância de cuidar da saúde mental da população idosa, independentemente do contexto da moradia e dos aspectos socioemocionais. Visto que, sintomas subclínicos da depressão estão associados aos comportamentais eliciados pelo CPF. Os resultados deste estudo indicam que o nível de escolaridade pode ser um fator protetor para o declínio das funções comportamentais relacionadas ao córtex pré-frontal em ambos os grupos (institucionalizados e não institucionalizados), enfatizando a importância da reserva cognitiva para a saúde mental de idosos.

Durante o processo de coleta de dados com os idosos encontraram-se algumas dificuldades, no que tange a aplicação dos protocolos de pesquisa em ambos os grupos, tendo em vista a quantidade de instrumentos utilizados. Diante do exposto, ressalta-se que ao fazer pesquisa envolvendo pessoas idosas faz-se necessário considerar o perfil desenvolvimetal da referida amostra e utilizar um procedimento sensível, indo além da coleta de dados.

Recomenda-se um olhar ativo desde a escolha dos instrumentos até o procedimento de aplicação dos mesmos. Faz-se necessário mais estudos desta magnitude que compreenda os sintomas comportamentais e a vunerabilidade do córtex préfrontal mediado por sintomas emocionais para aprimorar os conhecimentos teóricos e práticos que melhorem a qualidade de vida dos idosos. 


\section{Referências}

Alvarenga, M. R. M., Oliveira, M. A. de C., \& Faccenda, O. (2012). Sintomas depressivos em idosos: Análise dos itens da Escala de Depressão Geriátrica. Acta Paulista de Enfermagem, 25(4), 497-503.

Alves, M. B., Menezes, M. do R. de, Felzemburg, R. D. M., Silva, V. A. da, \& Amaral, J. B. do. (2017). Instituições de longa permanência para idosos: Aspectos físico-estruturais e organizacionais. Escola Anna Nery, 21. https://doi.org/10.1590/2177-9465-EAN-2016-0337.

Azevedo, L. M. de, Lima, H. H. G., Oliveira, K. S. A. de, Medeiros, K. F. de, Gonçalves, R. G., Nunes, V. M., \& Piuvesam, G. (2017). Perfil sociodemográfico e condições de saúde de idosos institucionalizados. Revista Brasileira de Pesquisa em Saúde/Brazilian Journal of Health Research, 19(3), $16-23$

Costa, T. N. M., Nieto, J. P. de S., Morikawa, L. S., Araújo, A. V. S. de, Cardoso, A. A. M., Mafra, B. G., Eiró, M., \& Costa, V. O. da. (2021). Análise do Mini Exame do estado mental de Folstein em idosos institucionalizados e não institucionalizados/ Analysis of Folstein's Mini State examination in institutionalized and non institutionalized elderly people. Brazilian Journal of Health Review, 4(2), 8319-8336. https://doi.org/10.34119/bjhrv4n2-357.

Brucki, S. M. D., Nitrini, R., Caramelli, P., Bertolucci, P. H. F., \& Okamoto, I. H. (2003). Sugestões para o uso do mini-exame do estado mental no Brasil. Arquivos de Neuro-Psiquiatria, 61(3B), 777-781. https://doi.org/10.1590/S0004-282X2003000500014

Ferreira, O. D. L., Barbosa, L. N. F., \& Alchieri, J. C. (2020). Validity Evidences of the Prefrontal Symptoms Inventory for the Elderly Brazilian Population. Clinics [online], 75 (e1863), 1-7. https://doi.org/10.6061/clinics/2020/e1863

Fonseca, L. M., Yokomizo, J. E., Bottino, C. M., \& Fuentes, D. (2016). Frontal Lobe Degeneration in Adults with Down Syndrome and Alzheimer's Disease: A Review. Dement Geriatr Cogn Disord. 2016;41(3-4):123-36. https://doi.org/10.1159/000442941

Formánek, T., Csajbók, Z., Wolfová, K., Kučera, M., Tom, S., Aarsland, D., \& Cermakova, P. (2020) Trajectories of depressive symptoms and associated patterns of cognitive decline. Sci Rep, 30;10(1):20888. 10.1038/s41598-020-77866-6.

Gerstenecker, A., Triebel, K., Eakin, A., Martin, R., \& Marson, D. (2018). Exploring the Factor Structure of Financial Capacity in Cognitively Normal and Impaired Older Adults. Clinical Gerontologist, 41(1), 33-41. https://doi.org/10.1080/07317115.2017.1387211

Jerez-Roig, J., de Medeiros, J. F., do Nascimento Moreira Fidélis, K., de Lima Filho, B. F., de Oliveira, N. P. D., Paes de Andrade, F. L. J., de Brito Macedo Ferreira, L. M., Leandro Bezerra Souza, D., \& Costa Lima, K. (2017). Activity Limitations in Brazilian Institutionalized Older Adults. Journal of Geriatric Physical Therapy, 40(4), 214-222. https://doi.org/10.1519/JPT.0000000000000101

Kaster, T., Daskalakis, Z., Noda, Y., Knyahnytska, Y., Downar, J., Rajji, T., \&Blumberger, D. M. (2018) Efficacy, tolerability, and cognitive effects of deep transcranial magnetic stimulation for late-life depression: a prospective randomized controlled trial. Neuropsychopharmacology: Official Publication of the American College of Neuropsychopharmacology, 43(11). https://doi.org/10.1038/s41386-018-0121-x

Kiyoshige, E., Kabayama, M., Gondo, Y., Masui, Y., Inagaki, H., Ogawa, M., Nakagawa, T., Yasumoto, S., Akasaka, H., Sugimoto, K., Ikebe, K., Arai, Y., Ishizaki, T., Rakugi, H., \& Kamide, K. (2019). Age group differences in association between IADL decline and depressive symptoms in community-dwelling elderly. BMC Geriatrics, 19(1), 309. https://doi.org/10.1186/s12877-019-1333-6

Klojčnik, M., Kavcic, V., \& Bakracevic Vukman, K. (2017). Relationship of Depression With Executive Functions and Visuospatial Memory in Elderly. International journal of aging \& human development, 85(4), 490-503. https://doi.org/10.1177/0091415017712186

Imaginario, C., Machado, P., Rocha, M., Antunes, C., \& Martins, T. (2017). Atividades de vida diária como preditores do estado cognitivo em idosos institucionalizados. Revista Portuguesa de Enfermagem de Saúde Mental,18, 37-43. https://doi.org/10.19131/rpesm.0190

Mohebbi, M., Agustini, B., Woods, R. L., McNeil, J. J., Nelson, M. R., Shah, R. C., Nguyen, V., Storey, E., Murray, A. M., Reid, C. M., Kirpach, B., Wolfe, R., Lockery, J. E., \& Berk, M. (2019). Prevalence of depressive symptoms and its associated factors among healthy community-dwelling older adults living in Australia and the United States. International Journal of Geriatric Psychiatry, 34(8), 1208-1216. https://doi.org/10.1002/gps.5119

Murayama, N., Ota, K., Matsunaga, Y., Ichikura, K., Fukase, Y., Tagaya, H., Iseki, E., \& Sato, K. (2019). Evaluating depression in cognitively healthy elderly people by using Mini-Mental State Examination. Psychogeriatrics, 20 (1), 96-103. https://doi.org/10.1111/psyg.12462

Organização Mundial da Saúde. Relatório sobre envelhecimento e saúde :Sociedade Brasileira de Geriatria e Gerontologia. (2015). https://sbgg.org.br/omsdivulga-relatorio-sobre-envelhecimento-e-saude/

Otte, C., Wingenfeld, K., Kuehl, L. K., Richter, S., Regen, F., Piber, D., \& Hinkelmann, K. (2015). Cognitive function in older adults with major depression: Effects of mineralocorticoid receptor stimulation. Journal of Psychiatric Research, 69, 120-125. https://doi.org/10.1016/j.jpsychires.2015.08.001

Pagotto, V., Silva, V. A. P., Pereira, L. V., \& Santos, D. P. M. A. (2016) Functionality comparison of elderly residing in two institutional modalities. Rev Eletr Enferm, 8, 1143. https://revistas.ufg.br/fen/article/view/34712/21177

Philippot, P., \& Agrigoroaei, S. (2017) Repetitive thinking, executive functioning, and depressive mood in the elderly. Aging Ment Health, 21(11):1192-1196. 10.1080/13607863.2016.1211619.

Ruiz-Sánchez de León, J. M., Pedrero-Pérez, E. J., Lozoya-Delgado, P., Llanero-Luque, M., Rojo-Mota, G., \& Puerta-García, C. (2012). Inventario de síntomas prefrontales para la evaluación clínica de las adicciones en la vida diaria: proceso de creación y propiedades psicométricas. Revista de Neurología, 54(11), 649-663. https://doi.org/10.33588/rn.5411.2012019

Santorelli, G., \& Ready, R. (2015). Alexithymia and Executive Function in Younger and Older Adults. The Clinical neuropsychologist, 29, 1-18. https://doi.org/10.1080/13854046.2015.1123296

Santos, A. A., Silva, A. L. S. B. da, Santos, F. A. B., Barbosa, J. E. S., Almeida, L. P. D. S., Mota, R. de S., \& Santos, A. C. N. dos. (2019). Functional and 
Research, Society and Development, v. 10, n. 10, e288101018705, 2021

(CC BY 4.0) | ISSN 2525-3409 | DOI: http://dx.doi.org/10.33448/rsd-v10i10.18705

cognitive profile of elderly residents in a long term care institution in the northeast'. Manual Therapy, Posturology \& Rehabilitation Journal, $17,681$.

Scherrer, G., Okuno, M. F. P., Oliveira, L. M. de, Barbosa, D. A., Alonso, A. C., Fram, D. S., \& Belasco, A. G. S. (2019). Qualidade de vida de idosos institucionalizados com e sem sinais de depressão. Revista Brasileira de Enfermagem, 72, 127-133. https://doi.org/10.1590/0034-7167-2018-0316

Steffens, D. C., Wang, L., Manning, K. J., \& Pearlson, G. D. (2017). Negative Affectivity, Aging, and Depression: Results From the Neurobiology of LateLife Depression (NBOLD) Study. The American Journal of Geriatric Psychiatry: Official Journal of the American Association for Geriatric Psychiatry, 25(10), 1135-1149. https://doi.org/10.1016/j.jagp.2017.03.017

Uehara, E., Charchat-Fichman, H., \& Landeira-Fernandez, J. (2013). Funções executivas: Um retrato integrativo dos principais modelos e teorias desse conceito. Neuropsicologia Latinoamericana, 5(3), 25-37. https://doi.org/10.5579/rnl.2013.145 